\section{Cahiers de Narratologie}

Analyse et théorie narratives

$10.2 \mid 2001$

La voix narrative

\title{
L'angoisse de la mort chez les différents personnages des Thibault de Roger Martin du Gard
}

\section{Maria Eugenia Peñalva Garcia}

\section{(2) OpenEdition}

1 Journals

Édition électronique

URL : http://journals.openedition.org/narratologie/10249

DOI : 10.4000/narratologie.10249

ISSN : 1765-307X

Éditeur

LIRCES

\section{Édition imprimée}

Date de publication : 1 janvier 2001

Pagination : 341-349

ISBN : 2914561032

ISSN : 0993-8516

\section{Référence électronique}

Maria Eugenia Peñalva Garcia, «L'angoisse de la mort chez les différents personnages des Thibault de Roger Martin du Gard », Cahiers de Narratologie [En ligne], 10.2 | 2001, mis en ligne le 01 janvier 2001, consulté le 11 juin 2020. URL : http://journals.openedition.org/narratologie/10249 ; DOI : https:// doi.org/10.4000/narratologie.10249 


\title{
L'ANGOISSE DE LA MORT CHEZ LES DIFFÉRENTS PERSONNAGES DES THIBAULT DE ROGER MARTIN DU GARD
}

\author{
Maria Eugenia PEÑALVA GARCIA \\ Université de Séville
}

Tout au long de sa vie, Martin du Gard sera confronté à la mort d'êtres chers. Lorsqu'il a 9 ans, il perd son arrièregrand-mère dont il était très proche. A cause de son jeune âge, cette épreuve lui cause un profond traumatisme.

Plus tard, il perdra beaucoup de nombreux êtres aimés, comme l'abbé Marcel Hebert, ses parents, son cousin Pierre Margaritis, son grand ami Gide et sa femme.

Lors de la naissance de sa fille, sa femme a failli perdre la vie, et après cette épreuve pénible, il écrit à son ami Bloch ${ }^{1}$ : «Ma fille a fait son apparition au milieu d'angoisses que je ne pourrai jamais oublier, et toute baignée de sueurs d'agonie ! Quel cauchemar! Et si douce que me soit aujourd'hui la paternité, vraiment j'ai failli la payer trop cher ».

La guerre de 1914 lui enlèvera un certain nombre d'amis comme Gustave Valmont, Fleury, Fernet et Quentin-Bouchart.

Toutes ces expériences ont construit une réelle obsession de la mort qui transparaît dans son œuvre littéraire.

La mort sera le thème central de ses trois premiers textes : Une vie de saint, Devenir! et L'Une de nous. Il écrira pendant la guerre que son besoin d'écrire lui permet de vaincre la mort en laissant quelque chose qui subsiste après lui au-delà de la mort. S'il a peur de mourir pendant la guerre de 1914, c'est parce qu'il ne laisserait derrière lui, qu'un seul texte important : Jean Barois. Dans ce premier roman, deux

1 Lettre de RMG à JRB, le 3 avril 1911, publié en Europe, septembre 1965 . 
grands thèmes chers à Martin du Gard s'expriment déjà : l'obsession de la mort et la perte de la foi catholique.

Mais c'est Les Thibault qui sera son œuvre principale et dont nous allons parler.

Martin du Gard meurt le vendredi 22 août 1958. Sur sa tombe au cimetière de Cimiez à Nice, sont gravés les mots suivants : «Aujourd'hui à moi, demain à toi ».

La peur de mourir sans rien laisser derrière lui a été une obsession constante. La publication et le succès des Thibault lui ont apporté une certaine tranquillité, voire même une certaine sérénité, avec la reconnaissance de l'importance de son œuvre. Il a écrit à Jean Delay, son ami : "La clé secrète de ma vie aura été l'horreur de l'oubli et de la mort $»^{2}$.

On constate que tous les personnages des Thibault n'ont pas le même statut pour Martin du Gard. Antoine et Oscar Thibault sont des personnages qui ne sont pas magnifiés par l'auteur, et ce, dans le sens qu'il renvoit leur existence dans l'aléatoire ; ainsi on connaît les moindres détails du personnage de Jacques bien avant son apparition dans le texte. Ces détails apparaissent depuis le début du premier récit, et on pourrait dire qu'ils servent de caisse de résonance à l'absence de Jacques, dont ils contribuent à forger le mythe.

Oscar Thibault est présenté comme un père autoritaire, très religieux, très exigeant à l'égard de ses enfants, et particulièrement de Jacques, ce qui génère une très mauvaise relation père-fils. L'approche de sa mort le choque énormément car il ne s'y attendait pas. Une fois qu'il comprend qu'il va mourir, il a très peur, et sa foi religieuse lui permettra de trouver la paix. A la fin de sa vie, Oscar est présenté comme un personnage mythique, c'est-à-dire qu'au-delà de la mort, il reste vivant dans la mémoire de ses enfants.

Beaucoup de critiques ont vu dans le personnage d'Antoine l'autoportrait de Martin du Gard. Il écrivit même

2 Jean DELAY : Introduction à la Correspondance Gide-Martin du Gard, p. 118. 

des Thibault de Roger Martin du Gard

dans son cahier de notes qu'il voulait faire d'Antoine et de Jacques les représentants fictifs de son cousin Pierre Margaritis, pour Jacques et de lui-même, pour Antoine. Mais quand il eut terminé son récit, il avoua qu'il avait mis de luimême dans les deux personnages des frères Thibault, même si Antoine était son porte-parole.

Antoine, donc, nous est présenté comme un médecin agnostique qui ne croit pas en Dieu et rejette la religion catholique ; il croit dans l'homme. Il agit envers Jacques avec paternalisme.

Jacques est présenté comme le personnage sensible et artistique du roman, c'est ce qui le rapproche de Martin du Gard. Il apparaît dès le début comme un personnage révolté qui refuse les lois paternelles, indépendant, idéaliste et capable d'aller jusqu'au bout de ses idéaux. Jacques est aimé de tout le monde y compris de son père ; d'ailleurs c'est le seul personnage autour duquel existe un tel consensus d'attachement. La plupart des autres personnages du roman, gravitent autour de Jacques. C'est le point de jonction des conflits qui surviennent au sein de cette famille; il fait le lien avec la famille Fontanin sur laquelle il exerce aussi une grande influence.

Antoine symbolise le héros qui accepte le monde tel qu'il est, acceptation au niveau social, politique et surtout au niveau des joies du corps.

Jacques, par contre, incarne le refus du monde; il ne se résigne ni aux fatalités ni aux limites corporelles, ni aux traditions sociales et politiques. Sa vie est une quête de l'absolu, de la pureté. En quelque sorte, Jacques est mystique et Antoine réaliste. C'est ce qui fait dire à Martin du Gard qu'ils occupent les deux pôles de sa propre personnalité. A la fin du roman, dans l'Epilogue, Antoine se rapprochera de son frère dans le sens qu'il le comprendra mieux que de son vivant, mais cette synthèse se réalise uniquement grâce à Antoine qui, après la guerre, a profondément changé.

Seuls les personnages masculins sont présentés comme des personnages mythiques, mais tous, féminins et masculins ont un rôle tragique. Leurs vies quotidiennes côtoient le drame : Martin du Gard décrit un monde sans issue, où les 
actions des hommes sont presque toutes condamnées, où les drames sont inévitables, où la finalité est le néant. Dans la famille Thibault, tous les personnages meurent, alors que chez les Fontanin seul le père décède, mais tous ont des destins tragiques. Jenny reste seule avec un enfant, et son grand amour est mort. Sa mère est veuve et Daniel est mutilé et décidé au suicide. La conscience de l'absurdité du monde pousse les héros de Martin du Gard à puiser au fond d'euxmêmes, suffisamment de force et de vitalité pour échapper au néant. C'est dans leur dimension tragique, mais aussi dans le sens de la solidarité humaine et de l'énergie que les héros de Martin du Gard rencontrent leur grandeur.

Cette mort qui le hante, occupe une place prépondérante dans Les Thibault. L'écriture permettait à l'auteur d'exorciser la peur de partir sans laisser de traces derrière lui.

L'architecture des Thibault se présente sous la forme très complexe d'une triple biographie familiale. Trois mouvements de longueur différente composent ce cycle polyphonique qui s'achèvera sur la mort de chacun des trois personnages de la famille Thibault. Parmi les nombreuses morts évoquées dans ce roman, nous aborderons uniquement celles de Jacques, d'Antoine et d'Oscar Thibault.

Dans la composition des personnages qui se trouvent face à la mort, deux éléments sont importants pour le romancier : d'abord le facteur du destin, et ensuite le facteur du caractère.

Les trois personnages ont un destin tragique par son issue fatale, mais chacun affrontera la situation de manière différente.

Oscar qui nous apparaît comme un homme très fort, autoritaire, orgueilleux, vivra une déchéance physique et mentale, jusqu'à la mort.

La maladie d'Oscar est décrite de façon impitoyable. La progression du cancer est évoquée au plan médical mais aussi au niveau de la psychologie du malade. Ignorant la réalité de son état de santé, il apprendra la vérité par une indiscrétion de M. Chasle. Martin du Gard projette sa propre terreur et son personnage criera à plusieurs reprises : «Oh! là ! là... 
L'angoisse de la mort chez les différents personnages des Thibault de Roger Martin du Gard

Oh! là ! là... J'ai peur... Je vais mourir, je vous dis ! Au secours! » (p. 267 M.P)

Martin du Gard a trouvé le ton juste, pour montrer avec émotion qu'un personnage arrogant, dur et autoritaire peut soudain devenir comme un enfant. Cette réaction est une réaction de l'âme, c'est une agonie psychique. L'écrivain lui apportera la sérénité en le rapprochant de la religion. L'abbé Vécard, après une conversation avec Oscar, quittera la chambre en disant à Antoine : "Entrez docteur, j'ai terminé » (p. 280 M.P.).

Oscar qui a trouvé le repos de l'âme, poursuivra son combat contre la douleur physique. Les douleurs ne seront apaisées qu'avec la morphine.

Les derniers jours, Oscar réfléchit sur sa vie avec sincérité et lucidité : ses bassesses avec Jacques, l'affection qu'il aurait pu lui donner. A la peur physique se substitue la peur métaphysique, celle de l'enfer ou du jugement dernier. Son retour à la religion l'aidera à trouver la paix et le pardon de Dieu.

Touché par la pénible agonie d'Oscar, Jacques lui pardonnera les attitudes rigides et autoritaires du passé. Il demande à son frère de faire le nécessaire pour abréger ces ultimes instants, si difficiles pour le corps et l'âme. Antoine consent à pratiquer l'injection libératrice.

Oscar décédé, l'auteur a voulu le revêtir d'une dimension symbolique, il devient plus imposant qu'autrefois.

L'acharnement de l'auteur à décrire les souffrances physiques du mourant s'explique par sa propre peur de la mort. Non pas de la mort en elle-même, mais de ce qui la précède : le corps qui ne nous appartient plus, qui est possédé par la maladie et abandonné aux soins de médecins, la souffrance. Roger Martin du Gard a éprouvé ses sentiments lorsque sa mère, morte d'un cancer, a vécu ces moments-là.

En mourant ainsi, malade, souffrant, incapable de se tenir debout, Oscar perd tout ce qui faisait son personnage : autorité, rigueur, respectabilité. Il devient une personne malade qui suscite la compassion, la tolérance, le pardon de son fils, et la sympathie du lecteur. 
Là encore, le goût de l'auteur pour le réalisme sert le texte et lui apporte une forte densité émotionnelle.

La mort de Jacques est bien différente, puisque engagé au service de son idéal, c'est un héros qui mourra. Comme son père, il appréhendait la mort. A Jenny qui l'accompagne lorsqu'ils sont témoins de la mort accidentelle d'un chien, il dira : «Ah, moi, presque sans interruption. Je veux dire que la plupart de mes pensées me ramènent à cette idée de la mort. » (I. 956)

L'adhésion de Jacques à la cause de l'Internationale Socialiste se justifie par son refus de la mort des innocents. Il comprend également le désir de son père de laisser au moins le nom d'Oscar Thibault à ses enfants. C'est un petit peu d'éternité et, donc, la négation du néant.

Si Jacques a peur de la mort, accomplir des actes exemplaires le séduit. Il s'investit alors complètement dans son projet, délaissant Jenny et tous les siens, et laissant le soin de l'organisation à son ami. Il a besoin de se sentir complètement possédé par son action, même si, et il en est conscient, sa mission est un peu suicidaire, il préfère n'en voir que l'aspect humanitaire.

Avant de monter dans l'avion qu'il prendra pour la dernière fois, Jacques s'imagine devant le conseil de guerre disant : "J'avais le choix entre un absurde sacrifice sur vos champs de bataille et le sacrifice dans la révolte, pour la libération de ceux que vous avez dupés. J'ai choisi ! j'ai accepté de mourir : mais pas à votre service ! Je meurs, parce que c'est l'unique moyen que vous m'avez laissé de lutter jusqu'au bout pour la seule chose qui continue à compter pour moi, en dépit de vos excitations à la haine : la fraternité entre les hommes. » (II, 721)

Cependant envisageant sa mort il aura aussi, comme son père, un instant d'épouvante, lorsqu'il est bien convaincu de sa mort prochaine, il dira: "la vie est l'unique bien. La sacrifier est fou. Le sacrifice est un crime, le crime contre nature! Tout acte d'héroïsme est absurde et criminel!" (II. p. 715) 
Rapidement il se maîtrise en pensant au succès de sa mission, à cet idéal qui justifie tout. A la fin, il pense que sa mort, même si sa mission échoue, sera un succès en elle-même : tout le monde comprendra son sacrifice et le présentera comme un exemple. Ce dernier aspect, auquel il n'avait pas pensé avant, ne lui est pas du tout indifférent et lui apporte finalement la paix.

Le romancier a structuré le récit de la mort de Jacques de la même façon que la mort du père. Jacques aura d'abord des souffrances psychiques, qu'il surmontera en trouvant la paix, mais il aura au moment de la mort plus de souffrances physiques que son père. Martin du Gard décrit avec réalisme une mort qui doit se rapprocher de ses expériences personnelles pendant la guerre de 1914. Certains critiques ont dit que c'était une mort ignoble, mais le romancier en avait besoin pour faire de Jacques un vrai héros. Sa mort a été exemplaire et utile, même pour Antoine, qui ne la comprenait pas au début. Jacques acquiert avec cette mort le statut de personnage mythique et rejoint ainsi la communauté des hommes exemplaires, même s'il les rejoint en solitaire. Il s'est sauvé luimême, en restant fidèle à sa personnalité et son idéal.

Antoine, lui, n'a jamais pensé à la mort. La mort de son père l'amènera à réfléchir à cet ultime instant de la vie. Antoine préfère l'action: "Vivre c'est agir, après tout! Méditer sur la vie? A quoi bon? » (II. 146)

Les gaz inhalés pendant la guerre, ont laissé des séquelles dont Antoine ne connaîtra la gravité que longtemps après. A son père, il avait caché la vérité, lui épargnant l'angoisse de la fin toute proche. Pour lui, il refuse la vérité aussi longtemps que possible. Avec la vérité, il connaîtra les souffrances psychiques, l'alternance des crises de désespoir et des moments de prostration. Fidèle à son tempérament, il évalue le temps nécessaire à l'évolution de la maladie. Il mettra ce temps à profit pour aider d'autres malades, écrire son journal et laisser des conseils éducatifs pour Jean-Paul.

Le récit de la mort d'Antoine est écrit avec la même méthode réaliste de description de la déchéance physique qui progresse jusqu'à la mort. A mesure que la mort avance, les annotations dans le journal sont plus courtes. Cette 
description de la mort d'Antoine est beaucoup moins impressionnante que les précédentes.

Antoine ne s'abandonne pas au désespoir comme l'a fait Oscar. Il ne se résigne pas, sa mort n'ayant aucun sens. Fuyant les pensées négatives, il se voue au déroulement de la guerre et aux souffrances d'autrui. Il conserve son optimisme et la maîtrise de ses pensées. Puis, les souffrances se font plus fortes, et son moral baisse. Dans son journal ne figurent plus que de notations médicales. Il ne songe jamais à s'appuyer sur un fondement religieux, il veut mourir comme il a vécu, dans l'agnosticisme. C'est ici qu'on trouve la pensée directe de Martin du Gard. Alors que les souffrances sont très pénibles, il décide finalement, comme il a fait pour son père, de se donner la mort à la morphine.

Roger Martin du Gard donne à chaque personnage une mort conforme à sa personnalité, il a respecté les trois Thibault et c'est là que réside le génie du créateur.

La mort, tout au long de cette œuvre, se présente comme le problème principal de la condition humaine. Presque tous les personnages de son roman vivent intensément et la seule pensée de la mort est inacceptable. Ils s'efforcent de dominer leur individualité quand ils essaient de réduire la signification de la mort. Néanmoins, chacun aura sa propre et singulière définition de la mort. Ils se sentent tellement accrochés à la vie, qu'ils ne se résignent pas à s'évanouir dans le néant.

Loin de considérer Martin du Gard comme un écrivain négatif, il faut voir en lui un grand optimiste grâce à la fin de son roman. Avant tout, on comprend son énorme humanité. 
L'angoisse de la mort chez les différents personnages

des Thibault de Roger Martin du Gard

\section{BIBLIOGRAFIA}

ABRAHAM (P.) : « Roger Martin du Gard », in Les lettres françaises, 28 août 1958.

Almeras (G.) : La Médecine dans Les Thibault de Roger Martin du Gard, Paris, P. Fournié et Cie, 1946.

ALlUIN (B.) : Martin du Gard. Romancier, Paris, Aux amateurs de livres, 1989.

BABCOK (A.) : «Perspective narrative dans Les Thibault », in Kentuchy Romance quaterly, $\mathrm{n}^{\circ} 1,1979$.

BARRUCAND (V.) : «A propos des médecins et de la médecine dans Les Thibault de Roger Martin du Gard », in Annales médico-psychologiques, 1966.

BILLY (A.) : « La fin des Thibault » in l'Euvre, 6 déc., 1936.

BOAK (D.) : Roger Martin du Gard, Oxford, Clarendon Pres, 1963.

CREMIEUX (B.) : «La Mort du Père, par Roger Martin du Gard », in Nouvelle Revue Française, 1er août 1929.

DEGRAEVE (D.) : " La figure du père dans Les Thibault », in Roman, 20/50, 12 déc, 1991.

FILIPOWSKA (I.) : Le Tragique de l'individu dans les romans de Roger Martin du Gard, Poznan, 1968.

GALLANT (M.) : Le Thème de la mort chez Roger Martin du Gard, Paris, Klincksieck, 1971.

MARTIN DU GARD (R.) : Euvres complètes, "Bibliothèque de la Pléiade », Préface d'Albert Camus, 2 vol., Paris, Gallimard, 1955.

VROLIJK (J.) : Le temps et la mort dans l'auvre romanesque de Roger Martin du Gard, thèse univers., dactyl., Paris, 1967. 